\title{
Editorial
}

\section{Whence Tendance? Accepting the Responsibility of Care for the Chronic Pain Patient}

Megan McGrew, DO ${ }^{1}$ and James Giordano, $\mathrm{PhD}^{2}$

Tendance: $n$. the act of attending and providing to needs or requirements; to offer care.

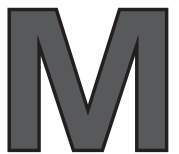

any patients present to the emergency room (ER) with a primary complaint of pain. Duchame and Barber (1), and Todd and colleagues (2) have shown that between onethird to one-half of all patients presenting to the ER reported chronic pain of severe intensity (i.e. mean pain scores $>8 / 10$ on the visual analog scale) that remained unrelieved at discharge in greater than $30 \%$ of cases. The progressive trend towards restricting longitudinal and/or multi-disciplinary care for chronic pain may be contributory, at least in part, to an increasing number of patients seeking pain relief through ER visits $(3,4)$. Thus, despite incentives of the congressionally declared Decade of Pain Control and Research and the National Pain Care Policy Act of 2003, recent estimates suggest that more than $70 \%$ of ER visits are related to chronic pain and/or its manifestations (5).

Dealing with chronic pain may be impacted by the situational and temporal constraints of the ER. Given that ER physicians typically spend an average of 12 to 15 minutes with (non-gravely injured) patients (6), it would be difficult to obtain sufficient past medical history, delve into concomitant psychological issues, inquire about prescription and non-prescription drug use, conduct a complete physical examination, and evaluate the nature and type of pain in such ways as to most capably provide the pain patient something other than "bridging therapeutics." Convention holds that the ER is simply not well equipped - with both resources and paradigmatically - to provide chronic pain care. Yet, for many under- or non-insured patients, the ER may represent a last or only option for treatment. Such issues become evermore problematic, given 1) restrictions in many employee's health care benefits due to current economic conditions, 2) discrepancy between insurance benefits provided and patients' long-term needs for managing chronic pain and its sequelae, and 3 ) increasing numbers of patients who have lost insurance coverage (for both primary and specialty pain care) due to unemployment and retirement.

The Emergency Medical Treatment and Labor Act (EMTALA) recognizes the need to treat pain as a symptom of emergency medical conditions, but not explicitly as a medical disorder itself (7). Thus, for many patients with chronic pain (syndromes), while exacerbated pain may be the impetus for seeking

From: ${ }^{1}$ Kansas City University of Medicine and Biosciences, Kansas City, MO; ${ }^{2}$ Institute for Psychological Sciences, University of Oxford, Oxford, UK; and Center for Neurotechnology Studies, Potomac Institute for Policy Studies, Arlington, VA.

Dr. McGrew is with the University of Medicine and Biosciences, Kansas City, MO.

Dr. Giordano is Professor of Neurophilosophy and Neuroethics, Institute for Psychological Sciences; Fellow, Blackfriar's Hall,

University of Oxford, St. Giles, Oxford, UK; and Director, Center for Neurotechnology Studies, Potomac Institute for Policy Studies, Arlington, VA.

Address correspondence: Prof. James Giordano Center for Neurotechnology Studies Potomac Institute for Policy Studies 901 N. Stuart St, Suite 900 Arlington, VA 22203

E-mail: james.giordano@bfriars.ox.ac.uk Disclaimer: There was no external funding in the preparation of this manuscript. 
emergency care, pain as a discrete medical disorder is not uniformly viewed (by physicians and hospital administrators) as justification for such intervention in the ER. Sandra Johnson has suggested a number of factors that may contribute to this view, including the prioritization of diagnosis over symptomatic relief, attitudinal biases regarding the gravity of chronic pain (as compared to other conditions characteristically seen in the ER), preconceived notions regarding patients' drug seeking (particularly when objective pathological findings are not readily revealed), and clinician anxieties regarding prescription of narcotic analgesics (8). In this latter regard, it should be noted that the Controlled Substance Act (CSA) was originally developed to guide and regulate the provision of scheduled compounds, including opioids, without impugning legitimate medical practice. Despite data suggesting that over the past 10 years, the Drug Enforcement Agency (DEA) has actually only pursued sanctions against less than one-tenth of one percent of licensed physicians (9), the climate instilled by the CSA and DEA has prompted Congressman Ron Paul to claim that "... the war on drugs is a war on doctors..." in which the ultimate victims are both physicians and inadequately treated pain patients (10).

Recently, we have conducted an informal survey of ER physicians in the greater Washington DC metropolitan area; approximately $80 \%$ opined that a chronic pain patient can be problematic in the ER setting, given the complexity of chronic pain, frequent physical and psychological co-morbidities, and multiple contingencies that often surround provision of opioids. However, less than $5 \%$ of ER physicians surveyed indicated that a pain service (or related neurology, psychiatry) consult is routinely called to assume management of such patients. One physician well summarized the situation, stating, "...the treat-or-triage system of the ER more often than not breaks down when dealing with chronic pain patients."

As John Hall and Mark Boswell discuss in this issue (11), there is considerable debate as to whether pain care is a constitutional right of all patients as persons. These authors astutely note that the provision of health care as a basic individual or social good does not necessarily guarantee complete delivery of all medical services to all patients all of the time. To be sure, there are several variables that constrain what services may be afforded to whom, and under what conditions. Often, the practicalities of clinical care are dictated by an alignment of patient, pathology, and medical specialty. There will be times when misalignment of these variables causes patients to fall between the proverbial cracks of the health care system. Clearly, the subjective and multidimensional aspects of chronic pain may complicate diagnosis and treatment, and may render its care in the ER (and perhaps anything other than the pain clinic) inadequate and/or inappropriate.

This speaks strongly to the putative barriers that have been proposed to impede sound and effective pain care, particularly outside of the auspices of pain medicine (12). Yet, John Peppin contends that these barriers may be tenuous, given the literature and current understanding of pain and its treatment resulting from the research efforts undertaken during the past 10 years' Decade of Pain Control and Research. Through the presentation of cases, Peppin illustrates that the problem of chronic pain, while conspicuous in the ER, is also evident within other clinical specialties (12). Thus, while Hall and Boswell (11) discuss the defeasibility of pain care as a right, we cannot deny that its provision is a fundamental and moral responsibility of the profession of pain medicine, and the practice of the pain physician. In this way, pain medicine should be seen as the logical and most ethically valid resource to be employed when treating the pain patient. We have argued that the "...technically right and morally good care of the pain patient is axiomatic to pain medicine" (13). We augment that claim by positing an equal obligation for other clinical specialties to engage pain physicians when treating any and all pain patients.

While prudent in principle, in reality this may not be possible given that many hospitals do not have (or have disbanded) a distinct pain service that is continuously available and on call to assist in or assume the sufficient diagnostics and management of chronic pain patients. The paucity of such services results in chronic pain patients' failure to receive apt or adequate care and may, in fact, contribute to their stigmatization, make access to care more difficult, and further heighten their vulnerability. Peppin claims that these factors marginalize the chronic pain patient. We agree, and add that such marginalization is antithetic to the claims of medicine as a healing and caring profession (12). Here we define care in the literal sense, as concern and heeding - concepts diametrically in opposition to any form of marginalization.

Pain care must be empowered within the culture of medicine, and such empowerment can only be sup- 
ported by economic and market values that sustain pain patients' access (and capacity to afford) such services. This will require significant change(s) in the current medical and economic systems. Without doubt, education plays a role in establishing the pathways for change. Perhaps all stakeholders involved in the care of pain patients must be provided with more comprehensive knowledge about diagnosis and treatment of chronic pain. But while this may mitigate attitudinal biases and foster sensitivity to the needs of those in pain, it would be meaningless unless the resources were available to implement the clinical diagnostics and therapeutics as required. So, perhaps a critical unanswered question at the end of this Decade of Pain Control and Research is not one of mechanism or effect, but rather how we will use the knowledge gained to develop and instill the ethically sound care of an increasing population of pain patients. We fear that failure to act upon what we have learned will only marginalize these patients further, and without adequate resources for responsible pain care, the ultimate question may be - whence tendance?

\section{References}

1. Duchame J, Barber C. A prospective blinded study on emergency pain assessment therapy. Jf Emergency Med 1995; 3:571-575.

2. Todd K, Sloan E, Chen C, Eder S, Wamstad K. Survey of pain etiology, management practices and patient satisfaction in two urban emergency departments. Canadian / Emergency Med 2002; 4:252-256.

3. Giordano J, Schatman, ME. A crisis in pain care- An ethical analysis. Part one: Facts, issues, and problems. Pain Physician 2008; 11:54-62.

4. Schatman ME. The demise of multidisciplinary pain management clinics? Prac Pain Management 2006; 6:30-41.
5. Disorbio J, Bruns D, Barolat G. Assessment and treatment of chronic pain: $A$ physician's guide to a biopsychosocial approach. Practical Pain Management 2006; 6:11-27.

6. Johnson S. The social, professional, and legal framework for the problem of pain management in emergency medicine. J Law, Medicine, Ethics 2005; 33:741-760.

7. Health and Human Services. Emergency Medical Treatment and Labor Act. 1986. www.cms.hhs.gov/emtala/

8. Johnson S. Pain management in the emergency department: Current landscape and agenda research. J Law, Medicine, Ethics 2005; 33:739-740.
9. Drug Enforcement Agency. www.usdoj. gov/dea/index.htm

10. Wilsey B, Fishman S, Ogden C. Prescription opioid abuse in the emergency department. J Law, Medicine, Ethics 2005; 33:770-782.

11. Hall J, Boswell MV. Ethics, law, and pain management as a patient right. Pain Physician 2009; 12:499-506.

12. Peppin J. The marginalization of chronic pain patients on chronic opioid therapy. Pain Physician 2009; 12:493-498.

13. Giordano J. Moral agency in pain medicine: philosophy, practice and virtue. Pain Physician 2006; 9:41-46. 
